\title{
Nowcasting with Google Trends
}

\author{
Yossi Matias \\ Google
}

Since launching Google Trends we have seen extensive interest in what can be learned from search trends. A plethora of studies have shown how to use search trends data for effective nowcasting in diverse areas such as health, finance, economics, politics and more.

We give an overview of Google Trends and Nowcasting, highlighting some exciting Big Data challenges, including large scale engineering, effective data analysis, and domain specific considerations.

An extended summary will be available at http://goo.gl/FbYh9

The original version of this chapter was revised: The copyright line was incorrect. This has been corrected. The Erratum to this chapter is available at DOI: 10.1007/978-3-319-02432-5_33

O. Kurland, M. Lewenstein, and E. Porat (Eds.): SPIRE 2013, LNCS 8214, p. 4, 2013.

(C) Springer-Verlag Berlin Heidelberg 2013 